\title{
Knowing you beyond race: the importance of individual feature encoding in the other-race effect
}

\author{
JenniferT. Kubota ${ }^{1 *}$ and Keith B. Senholzi ${ }^{2}$ \\ Phelps Lab, Department of Psychology, New York University, New York, NY, USA \\ CU Social Neuroscience Lab, Department of Psychology, University of Colorado Boulder, Boulder, CO, USA \\ *Correspondence: jk149@nyu.edu
}

\section{A commentary on}

Why some faces won't be remembered: brain potentials illuminate successful versus unsuccessful encoding for same-race and other-race faces

by Lucas, H. D., Chiao, J. Y., and Paller K. A. (2011). Front. Hum. Neurosci. 5:20. doi: 10.3389/fnhum.2011.00020

The common phrase, "they all look alike to me" is a robustly supported empirical finding known as the other-race effect (ORE; see Malpass and Kravitz, 1969). The ORE is a memory bias indexed by superior memory for ingroup members of the perceiver's race relative to outgroup members of a different race. On the surface, the ORE may seem harmless, but this deficit holds devastating consequences, such as inaccuracies in eyewitness testimony and resultant wrongful convictions (Brigham and Ready, 2005). Empirical evidence suggests that failure to recognize outgroup members arises partially due to failures during encoding. But the question remains, what precisely do people fail to encode about other-race (OR) faces? Lucas et al. (2011) posed this question in a recent investigation. The authors' findings suggest that people fail to recognize outgroup members because they are insensitive to the individual features of their faces, features that would otherwise aid in the discrimination of group members. These data build upon current knowledge of face-race perception by considering the neural processes that underlie encoding failures of outgroup members and raise critical questions regarding the ORE, individuation, and the extent to which face processing may be modulated to encourage deeper encoding.

To investigate this phenomenon, Lucas et al. (2011) conducted an experiment comprised of two critical phases. In phase one, participants studied faces that were either ingroup, same-race (SR) faces, or outgroup, OR faces. During phase two, recognition was tested through presentation of old, studied faces, and new, unstudied faces. Participants judged if they had previously seen each face. Event-related brain potentials, a measure of neural electrical activity, were recorded during the study phase and compared between faces subsequently remembered and those forgotten. Memory was greater for SR faces compared with OR faces, replicating the ORE. In a novel demonstration, neural activity during the study phase resulted in several time points in processing that were sensitive to encoding differences for SR versus OR faces. An occipito-temporal deflection in ERPs, the P2, and a frontocentral deflection, the N200, varied as a function of the ORE between 200 and $250 \mathrm{~ms}$ after viewing a face. These findings represent an innovative method for investigating factors that give rise to the ORE by comparing neural processing during face learning for $\mathrm{OR}$ faces later remembered and forgotten.

An interesting extension of Lucas et al.'s (2011) findings is the consideration of whether attention to unique features of faces encourages deeper encoding of individuating traits and behaviors of outgroup members. In the social psychological literature, individuation is defined as the application of unique information to a person rather than stereotypes. Individuation is thought to involve effortful, complex attribute analysis of person-specific information occurring over an extended period of time (Fiske and Neuberg, 1990). The authors' research intimates that there may exist foundational processes that support individuation within milliseconds of viewing an individual. This hypothesized relationship is in line with previous work demonstrating that ERPs indexing early category processing predict later category judgments (Kubota and Ito, 2007). Individual feature encoding can therefore set the stage for individualized impression formation, just as category-based feature encoding can lead to category-based impressions. Thus, whether categorical or individuated processing dominates impressions might differ as a function of perceivers' early attention. Future research may do well to investigate the relationship between feature individuation (Lucas et al., 2011) and individuation in the context of impression formation.

One reason perceivers apply stereotypes is to maintain cognitive efficiency. Fiske and Taylor (1984) coined the term "cognitive miser" to refer to this simple and efficient means of information processing. By stereotyping, people are able to minimize time and effort getting to know individuals (which presumably occurs through individuated processing), but still retain the perception that they know something about them from group-based information. Interestingly, when people are motivated, they individuate targets (e.g., Fiske and Neuberg, 1990). Therefore, increasing a perceiver's motivation through simple instructions may be sufficient to trigger an immediate individuated processing approach to outgroup members and should increase both individuation in behavioral responding and attention to individual features (Hugenberg et al., 2007). Giving instructions to individuate also diminishes activity in neural regions involved in category-based processing and increases activation in regions involved in empathy and perspective-taking (e.g., Harris and Fiske, 2006; Ames et al., 2008). If this early increased attention to individualized features spurs downstream individualized impressions, implementing manipulations to increase it may serve as a straightforward solution to guide personalized impression formation and increased recognition of outgroup faces.

Lucas et al. (2011) contribute to the growing literature on how memory differences for individuals of ORs are shaped by social and perceptual factors during neural processing. These results likely extend beyond racial-group membership; recent evidence indicates that recognition deficits are found for members of outgroups 
independent of race. Bernstein et al. (2007) demonstrated that merely categorizing someone on university affiliation leads to memory deficits. If the socio-cognitive factors that underlie race-based and group-based memory deficits are shared, manipulations to increase memory, and personalized processing of racial outgroup members should function analogously for more broadly defined outgroups.

\section{REFERENCES}

Ames, D. L., Jenkins, A. C., Banaji, M. R., and Mitchell, J. P. (2008). Taking another person's perspective increases self-referential processing. Psychol. Sci. 19, 642-644.

Bernstein, M. J., Young, S. G., and Hugenberg, K. (2007). The cross-category effect: mere social categorization is sufficient to elicit an own-group bias in face recognition. Psychol. Sci. 18, 709-712.
Brigham, J. C., and Ready, D. J. (2005). Own-race bias in lineup construction. Law Hum. Behav. 9, 415-424.

Fiske, S. T., and Neuberg, S. L. (1990). "A continuum model of impression formation from category-based to individuated processes: influences of information and motivation on attention and interpretation," in Advances in Experimental Social Psychology, Vol. 3, ed. M. P. Zanna (New York: Academic Press), 1-74.

Fiske, S. T., and Taylor, S.E. (1984). Social Cognition. New York: Random House.

Harris, L. T., and Fiske, S. T. (2006). Dehumanizing the lowest of the low: neuroimaging responses to extreme outgroups. Psychol. Sci. 17, 847-853.

Hugenberg, K., Miller, J., and Claypool, H. M. (2007). Categorization and individuation in the cross-race recognition deficit: toward a solution to an insidious problem. J. Exp. Soc. Psychol. 43, 334-340.

Kubota, J. T., and Ito, T. A. (2007). Multiple cues in social perception: the time course of processing race and facial expression. J. Exp. Soc. Psychol. 43, 738-752.
Lucas, H. D., Chiao, J. Y., and Paller, K. A. (2011). Why some faces won't be remembered: brain potentials illuminate successful versus unsuccessful encoding for same-race and other-race faces. Front. Hum. Neurosci. 5:20. doi: 10.3389/ fnhum.2011.00020

Malpass, R. S., and Kravitz, J. (1969). Recognition for faces of own and other race. J. Pers. Soc. Psychol. 13, 330-334.

Received: 14 March 2011; accepted: 15 March 2011; published online: 28 March 2011.

Citation: Kubota JT and Senholzi KB (2011) Knowing you beyond race: the importance of individual feature encoding in the other-race effect. Front. Hum. Neurosci. 5:33. doi: 10.3389/fnhum.2011.00033

Copyright $\odot 2011$ Kubota and Senholzi. This is an openaccess article subject to an exclusive license agreement between the authors and Frontiers Media SA, which permits unrestricted use, distribution, and reproduction in any medium, provided the original authors and source are credited. 